\title{
The Hilbert-Type Integral Inequality with the System Kernel of $-\lambda$ Degree Homogeneous Form
}

\section{ZiTIAN XIE*}

Dept. of Math. Zhaoqing University, Zhaoqing, Guangdong, 526061, P. R. China e-mail : gdzqxzt@163.com

\section{ZHENG ZENG}

Shaoguan University, Shaoguan, Guangdong, China 512005

e-mail : zz@sgu.edu.cn

Abstract. In this paper, the integral operator is used. We give a new Hilbert-type integral inequality, whose kernel is the homogeneous form with degree $-\lambda$ and with three pairs of conjugate exponents and the best constant factor and its reverse form are also derived. It is shown that the results of this paper represent an extension as well as some improvements of the earlier results.

\section{Introduction}

If $p>1, \frac{1}{p}+\frac{1}{q}=1, f \in L^{p}(0, \infty)$, and $g \in L^{q}(0, \infty), f(x), g(x) \geq 0$, such that $0<\int_{0}^{\infty} f^{p}(x) d x<\infty$ and $0<\int_{0}^{\infty} g^{q}(x) d x<\infty$, then [1]:

$$
\int_{0}^{\infty} \int_{0}^{\infty} \frac{f(x) g(y)}{x+y} d x d y<\frac{\pi}{\sin (\pi / p)}\left\{\int_{0}^{\infty} f^{p}(x) d x\right\}^{1 / p}\left\{\int_{0}^{\infty} g^{q}(x) d x\right\}^{1 / q}
$$

where the constant factor $\frac{\pi}{\sin (\pi / p)}$ is the best possible.

Inequality (1.1) is named Hardy-Hilbert's integral inequality, which is important in analysis and applications. It has been studied and generalized in many directions by a number of people [2-21].

Let $(T f)(y):=\int_{0}^{\infty} H(x, y) f(x) d x,\|f\|_{p}:=\left(\int_{0}^{\infty}|f(x)|^{p}\right)^{1 / p}$. We have $(T f)(g):=$ $\int_{0}^{\infty}\left(\int_{0}^{\infty} H(x, y) f(x) d x\right) g(y) d y$. If $H(x, y)=\frac{1}{x+y}$, in 2006,Yang [2] rewrote (1.1) as

$$
(T f)(g)<\frac{\pi}{\sin (\pi / p)}\|f\|_{p}\|g\|_{q}
$$

where $T: L^{r}(0, \infty) \rightarrow L^{r}(0, \infty)(r=p, q)$ is an integral operator.

For the purposes, we introduce some notations as follows:

* Corresponding Author.

Received September 7, 2009; accepted January 28, 2010.

2000 Mathematics Subject Classification: 26D15.

Key words and phrases: Integral operator; Hilbert-type integral inequality, weight function, Hölder's inequality. 
Let $p>0, p \neq 1 \frac{1}{p}+\frac{1}{q}=1, r>1, \frac{1}{r}+\frac{1}{s}=1$. A norm of $f$ with the weight function $w(x)$ is defined by

$$
\|f\|_{p, w}:=\left\{\int_{0}^{\infty} w(x)|f(x)|^{p} d x\right\}^{1 / p} .
$$

where $f(x), w(x) \geq 0$ are measurable functions defined on $(0, \infty)$. If $\|f\|_{p, w}<\infty$, then it is marked by $f \in L_{w}^{p}\left(\mathbb{R}_{+}\right)$.

Supposing that $H(x, y) \geq 0$ is a real measurable in $(0, \infty) \times(0, \infty)$ and satisfies $H(u x, u y)=u^{-\lambda} H(x, y)(\lambda>0, u>0)$ for $(x, y) \in(0, \infty) \times(0, \infty)$, then $H(x, y)$ is called a homogeneous function of $-\lambda$-degree. Then we have the formal inner as follows:

$$
(T f, g)=(T g, f)=\int_{0}^{\infty}\left(\int_{0}^{\infty} H(x, y) f(x) d x\right) g(y) d y=\int_{0}^{\infty} \int_{0}^{\infty} H(x, y) f(x) g(y) d x d y .
$$

Define the integral operator $T$ as:

for $f \in L^{p}(0, \infty)$,

$$
(T f)(y):=\int_{0}^{\infty} H(x, y) f(x) d x, y \in(0, \infty)
$$

or for $g \in L^{q}(0, \infty)$,

$$
(T g)(x):=\int_{0}^{\infty} H(x, y) g(y) d y, x \in(0, \infty)
$$

where $T$ is call Hilbert-type integral operator, if $T$ is bounded. $H(x, y)$ is call the kernel of $T$.

The main objective of this paper is to build a new Hilbert-type integral inequality, whose Kernel is the homogeneous form degree $-\lambda$ with three pairs of conjugate exponents and with the best constant factor. As applications, the equivalent forms and some particular results are given.

In the following, we always suppose that

1) $t \geq 0, p>0, p \neq 1 \frac{1}{p}+\frac{1}{q}=1 . r>1, \frac{1}{r}+\frac{1}{s}=1 . l>1, \frac{1}{l}+\frac{1}{k}=1$.

2) $H(x, y) \geq 0$ is a real measurable in $(0, \infty) \times(0, \infty)$ homogeneous function of $-\lambda$-degree $(\lambda>0)$, and $0<\int_{0}^{\infty} H(1, \sigma) \sigma^{-1+t-\frac{2 t h-s \lambda}{s h}} d \sigma<\infty$.

3) $w(x)=x^{p\left(1-t+\frac{2 t l-r \lambda}{r l}\right)-1}, \hat{w}(x)=x^{q\left(t-\frac{2 t l-r \lambda}{r l}\right)-1} ; \widetilde{w}(y)=y^{q\left(1-t+\frac{2 t h-s \lambda}{s h}\right)-1}, \bar{w}(y)=$ $y^{p\left(t-\frac{2 t h-s \lambda}{s h}\right)-1}$.

\section{Lemma and main results}

Lemma. Define the weight functions:

$$
W(x)=\int_{0}^{\infty} H(x, y) \frac{x^{\frac{p}{q}\left(1-t+\frac{2 t l-r \lambda}{r l}\right)}}{y^{1-t+\frac{2 t h-s \lambda}{s h}}} d y, \quad \widetilde{W}(y)=\int_{0}^{\infty} H(x, y) \frac{y^{\frac{q}{p}\left(1-t+\frac{2 t h-s \lambda}{s h}\right)}}{x^{1-t+\frac{2 t l-r \lambda}{r l}}} d x .
$$


then

$$
W(x)=K_{1} w(x), \quad \widetilde{W}(y)=K_{2} \widetilde{w}(y)
$$

where

$$
K_{1}=\int_{0}^{\infty} H(1, \sigma) \sigma^{-1+t-\frac{2 t h-s \lambda}{s h}} d \sigma, \quad K_{2}=\int_{0}^{\infty} H(1, \sigma) \sigma^{1-t+\frac{2 t l-r \lambda}{r l}-2+\lambda} d \sigma
$$

and $K:=K_{1}=K_{2}$.

Proof. Setting $y=x \sigma$ we have $W(x)=K_{1} w(x)$.

on the other hand, we have

$$
\widetilde{W}(y)=\int_{0}^{\infty} H\left(\frac{y}{\sigma}, y\right) \frac{y^{\frac{q}{p}\left(1-t+\frac{2 t h-s \lambda}{s h}\right)}}{\left(\frac{y}{\sigma}\right)^{1-t+\frac{2 t l-r \lambda}{r l}}} d\left(\frac{y}{\sigma}\right)=K_{2} \widetilde{w}(y)
$$

easily $K_{1}=K_{2}=K$. the lemma is proved.

Theorem 2.1. If $p>1, f \in L_{w}^{p}\left(\mathbb{R}_{+}\right), g \in L_{\widetilde{w}}^{q}\left(\mathbb{R}_{+}\right)$, and $\|f\|_{p, w}>0,\|g\|_{q, \widetilde{w}}>0$, then

$$
(T f, g)=(T g, f)=\int_{0}^{\infty} \int_{0}^{\infty} H(x, y) f(x) g(y) d x d y<K\|f\|_{p, w}\|g\|_{q, \widetilde{w}} ;
$$

If $f \in L_{w}^{p}\left(\mathbb{R}_{+}\right)$and $\|f\|_{p, w}>0$, then $T f \in L_{\bar{w}}^{p}\left(\mathbb{R}_{+}\right)$and

$$
\|T f\|_{p, \bar{w}}=\left\{\int_{0}^{\infty} y^{p\left(t-\frac{2 t h-s \lambda}{s h}\right)-1}\left(\int_{0}^{\infty} H(x, y) f(x) d x\right)^{p} d y\right\}^{\frac{1}{p}}<K^{p}\|f\|_{p, w} .
$$

$K$ is defined by lemma, both constant factors, $K$ and $K^{p}$ are the best possible and inequalities (2.1) and (2.2) are equivalent.

Theorem 2.2. If $1>p>0, f(x), g(x) \geq 0$, such that $f \in L_{w}^{p}\left(\mathbb{R}_{+}\right), g \in L_{\widetilde{w}}^{q}\left(\mathbb{R}_{+}\right)$, and $\|f\|_{p, w}>0,\|g\|_{q, \widetilde{w}}>0$, then

$$
(T f, g)=\int_{0}^{\infty} \int_{0}^{\infty} H(x, y) f(x) g(y) d x d y>K\|f\|_{p, w}\|g\|_{q, \widetilde{w}} .
$$

If $f \in L_{w}^{p}\left(\mathbb{R}_{+}\right)$and $\|f\|_{p, w}>0$, then $T f \in L_{\bar{w}}^{p}\left(\mathbb{R}_{+}\right)$and

$$
\|T f\|_{p, \bar{w}}=\left\{\int_{0}^{\infty} y^{p\left(t-\frac{2 t h-s \lambda}{s h}\right)-1}\left(\int_{0}^{\infty} H(x, y) f(x) d x\right)^{p} d y\right\}^{\frac{1}{p}}>K^{p}\|f\|_{p, w},
$$

where both constant factors $K$ and $K^{p}$ are the best possible and inequalities (2.3) and (2.4) are equivalent.

We prove only Theorem 2.2, since the proof of Theorem 2.1 is the similar. 
Proof of Theorem 2.2 By Hölder's inequality [22] and results of lemma we have,

$$
\begin{gathered}
(T f, g)=\int_{0}^{\infty} \int_{0}^{\infty} H(x, y) f(x) \frac{x^{\frac{1}{q}\left(1-t+\frac{2 t l-r \lambda}{r l}\right)}}{y^{\frac{1}{p}\left(1-t+\frac{2 t h-s \lambda}{s h}\right)}} g(y) \frac{y^{\frac{1}{p}\left(1-t+\frac{2 t h-s \lambda}{s h}\right)}}{x^{\frac{1}{q}\left(1-t+\frac{2 t l-r \lambda}{r l}\right)}} d x d y \\
\geq\left\{\int_{0}^{\infty} \int_{0}^{\infty} H(x, y) \frac{x^{\frac{p}{q}\left(1-t+\frac{2 t l-r \lambda}{r l}\right)}}{y^{1-t+\frac{2 t h-s \lambda}{s h}}} f^{p}(x) d y d x\right\}^{\frac{1}{p}} \\
\left.\qquad \int_{0}^{\infty} \int_{0}^{\infty} H(x, y) \frac{y^{\frac{q}{p}\left(1-t+\frac{2 t h-s \lambda}{s h}\right)}}{x^{1-t+\frac{2 t l-r \lambda}{r l}}} g^{q}(y) d x d y\right\}^{\frac{1}{q}} \\
=\left\{\int_{0}^{\infty} W(x) f^{p}(x) d x\right\}^{\frac{1}{p}}\left\{\int_{0}^{\infty} \widetilde{W}(y) g^{q}(y) d y\right\}^{\frac{1}{q}}=K\|f\|_{p, w}\|g\|_{q, \widetilde{w}} .
\end{gathered}
$$

If (2.5) takes the form of equality, then there exist constants $M$ and $N$, such that they are not all zero and

$$
M \frac{x^{\frac{p}{q}\left(1-t+\frac{2 t l-r \lambda}{r l}\right)}}{y^{1-t+\frac{2 t h-s \lambda}{s h}}} f^{p}(x)=N \frac{y^{\frac{q}{p}\left(1-t+\frac{2 t h-s \lambda}{s h}\right)}}{x^{1-t+\frac{2 t l-r \lambda}{r l}}} g^{q}(y) \quad \text { a.e. in }(0, \infty) \times(0, \infty) .
$$

Hence there exists a constant $C$, such that

$$
M x^{p\left(1-t+\frac{2 t l-r \lambda}{r l}\right)} f^{p}(x)=N y^{q\left(1-t+\frac{2 t h-s \lambda}{s h}\right)} g^{q}(y)=C \quad \text { a.e. in }(0, \infty),
$$

Without loss of generality, suppose that $M \neq 0$, we my get $x^{p\left(1-t+\frac{2 t l-r \lambda}{r l}\right)-1} f^{p}(x)=$ $C /(M x)$ a.e. in $(0, \infty)$ which contradicts $f \in L_{w}^{p}\left(\mathbb{R}_{+}\right)$. Hence $(2.6)$ takes a strict inequality and we have (2.3).

If the constant factor $K$ in (2.3) is not the best possible, then there exists a positive constant $\widetilde{K}$ (with $\widetilde{K}>K$ ), such that (2.3) is still valid if we replace $K$ by $\widetilde{K}$. Setting $f_{n}$ and $g_{n}$ as; $f_{n}(x)=g_{n}(x)=0$, for $x \in(0,1) ; f_{n}(x)=$ $x^{-1+t-\frac{2 t l-r \lambda}{r l}-\frac{1}{n p}}, g_{n}(x)=x^{-1+t-\frac{2 t h-s \lambda}{s h}-\frac{1}{n q}}$, for $x \in[1, \infty)$. then for $n \in \mathbb{N}$,

$$
\left(T f_{n}, g_{n}\right)>\widetilde{K}\left\|f_{n}\right\|_{p, w}\left\|g_{n}\right\|_{q, \widetilde{w}}=n \widetilde{K} .
$$

Setting $x=\frac{y}{\sigma}$, then we obtain

$$
\begin{aligned}
J_{n}:= & \left(T f_{n}, g_{n}\right)=\int_{0}^{\infty} \int_{0}^{\infty} H(x, y) f_{n}(x) g_{n}(y) d x d y \\
= & \int_{1}^{\infty} \int_{1}^{\infty} H(x, y) x^{-1+t-\frac{2 t l-r \lambda}{r l}-\frac{1}{n p}} y^{-1+t-\frac{2 t h-s \lambda}{s h}-\frac{1}{n q}} d x d y \\
= & \int_{1}^{\infty} y^{-1-\frac{1}{n}}\left(\int_{0}^{y} H(1, \sigma) \sigma^{1-t+\frac{2 t l-r \lambda}{r l}-2+\lambda+\frac{1}{n p}} d \sigma\right) d y \\
=\int_{1}^{\infty} y^{-1-\frac{1}{n}} & \left(\int_{0}^{1} H(1, \sigma) \sigma^{-1+t-\frac{2 t h-s \lambda}{s h}+\frac{1}{n_{p}}} d \sigma\right) d y \\
& \quad+\int_{1}^{\infty} y^{-1-\frac{1}{n}}\left(\int_{1}^{y} H(1, \sigma) \sigma^{-1+t-\frac{2 t h-s \lambda}{s h}+\frac{1}{n_{p}}} d \sigma\right) d y
\end{aligned}
$$




$$
\begin{aligned}
& =n \int_{0}^{1} H(1, \sigma) \sigma^{-1+t-\frac{2 t h-s \lambda}{s h}+\frac{1}{n p}} d \sigma \\
& \quad+\int_{1}^{\infty} H(1, \sigma) \sigma^{-1+t-\frac{2 t h-s \lambda}{s h}+\frac{1}{n p}}\left(\int_{\sigma}^{\infty} y^{-1-\frac{1}{n}} d y\right) d \sigma \\
& =n\left[\int_{0}^{1} H(1, \sigma) \sigma^{-1+t-\frac{2 t h-s \lambda}{s h}+\frac{1}{n p}} d \sigma+\int_{1}^{\infty} H(1, \sigma) \sigma^{-1+t-\frac{2 t h-s \lambda}{s h}-\frac{1}{n q}} d \sigma\right] .
\end{aligned}
$$

In view of $(2.6)$, we have $n \widetilde{K}<J_{n}$.

Secondly, by Fatou lemma, one has

$$
\begin{aligned}
& \widetilde{K} \leq \min _{n \rightarrow \infty} \frac{1}{n} J_{n} \\
& =\min _{n \rightarrow \infty}\left[\int_{0}^{1} H(1, \sigma) \sigma^{-1+t-\frac{2 t h-s \lambda}{s h}+\frac{1}{n_{p}}} d \sigma+\int_{1}^{\infty} H(1, \sigma) \sigma^{-1+t-\frac{2 t h-s \lambda}{s h}-\frac{1}{n q}} d \sigma\right] \\
& \leq\left[\int_{0}^{1} \frac{\min _{n \rightarrow \infty}}{n} H(1, \sigma) \sigma^{-1+t-\frac{2 t h-s \lambda}{s h}+\frac{1}{n p}} d \sigma+\int_{1}^{\infty} \min _{n \rightarrow \infty} H(1, \sigma) \sigma^{-1+t-\frac{2 t h-s \lambda}{s h}-\frac{1}{n q}} d \sigma\right] \\
& =K \text {. }
\end{aligned}
$$

It follows that $\widetilde{K} \leq K$, which contradicts the fact that $K<\widetilde{K}$. Hence the constant $K$ in $(2.3)$ is the best possible.

For $x>0, n \in \mathbb{N}$, setting a bounded measurable function $\widetilde{f}_{n}$ as

$$
\widetilde{f}_{n}(x)=\left\{\begin{array}{l}
n, \quad \text { if } f(x)>n, \\
f(x), \text { if } \frac{1}{n} \leq f(x) \leq n, \\
\frac{1}{n}, \quad \text { if } f(x)<\frac{1}{n},
\end{array}\right.
$$

by the condition of $f \in L_{w}^{p}\left(\mathbb{R}_{+}\right)$, there exists $n_{0} \in \mathbb{N}$, such that for $n \geq n_{0} \in \mathbb{N}, 0<$ $\int_{1 / n}^{n} w(x) \widetilde{f}(x)^{p} d x<\infty$. Setting $\widetilde{g}_{n}(y)=y^{p\left(t-\frac{2 t h-s \lambda}{s h}\right)-1}\left(\int_{1 / n}^{n} H(x, y) \widetilde{f}_{n}(x) d x\right)^{p-1},\left(\frac{1}{n}<\right.$ $x \leq n ; n \geq n_{0}$ ) by Hölder's inequality [22], we have

$$
\begin{aligned}
\infty & >\int_{1 / n}^{n} y^{q\left(1-t+\frac{2 t h-s \lambda}{s h}\right)-1} \widetilde{g}_{n}^{q}(y) d y \\
& =\int_{1 / n}^{n} y^{p\left(t-\frac{2 t h-s \lambda}{s h}\right)-1}\left(\int_{1 / n}^{n} H(x, y) \widetilde{f}_{n}(x) d x\right)^{p} d y \\
& =\int_{1 / n}^{n} \int_{1 / n}^{n} y^{p\left(t-\frac{2 t h-s \lambda}{s h}\right)-1}\left(\int_{1 / n}^{n} H(x, y) \widetilde{f}_{n}(x) d x\right)^{p} d x d y \\
& =\int_{1 / n}^{n} \int_{1 / n}^{n} H(x, y) \widetilde{f}_{n}(x) \widetilde{g}_{n}(y) d x d y \\
& >K\left(\int_{1 / n}^{n} x^{p\left(1-t+\frac{2 t l-r \lambda}{r l}\right)-1} \widetilde{f}_{n}^{p}(x) d x\right)^{1 / p}\left(\int_{1 / n}^{n} y^{q\left(1-t+\frac{2 t h-s \lambda}{s h}\right)-1} \widetilde{g}_{n}(y) d y\right)^{1 / q} .
\end{aligned}
$$




$$
0<K^{p} \int_{1 / n}^{n} x^{p\left(1-t+\frac{2 t l-r \lambda}{r l}\right)-1} \widetilde{f}_{n}^{p}(x) d x<\int_{1 / n}^{n} y^{q\left(1-t+\frac{2 t h-s \lambda}{s h}\right)-1} \widetilde{g}_{n}^{q}(y) d y<\infty
$$

For $n \rightarrow \infty$, if $\int_{0}^{\infty} y^{q\left(1-t+\frac{2 t h-s \lambda}{s h}\right)-1} \widetilde{g}_{\infty}^{q}(y) d y=\infty$, then we have (2.4); if $0<$ $\int_{0}^{\infty} y^{q\left(1-t+\frac{2 t h-s \lambda}{s h}\right)-1} \widetilde{g}_{\infty}^{q}(y) d y<\infty$, then by using (2.3), both (2.7) and (2.8) still take the form of strict inequalities, and we have (2.4).

On the other hand, if inequality (2.4) holds, then by Hölder's inequality, we have

$$
\begin{gathered}
(T f, g)=\int_{0}^{\infty} \int_{0}^{\infty} H(x, y) f(x) g(y) d x d y \\
=\int_{0}^{\infty}\left(y^{-1+t-\frac{2 t h-s \lambda}{s h}+\frac{1}{q}} \int_{0}^{\infty} H(x, y) f(x) d x\right)\left(y^{1-t+\frac{2 t h-s \lambda}{s h}-\frac{1}{q}} g(y)\right) d y \\
\geq\left\{\int_{0}^{\infty} y^{p\left(t-\frac{2 t h-s \lambda}{s h}\right)-1}\left(\int_{0}^{\infty} H(x, y) f(x) d x\right)^{p} d y\right\}^{\frac{1}{p}} \\
\left\{\int_{0}^{\infty} y^{q\left(1-t+\frac{2 t h-s \lambda}{s h}\right)-1} g(y) d y\right\}^{\frac{1}{q}}
\end{gathered}
$$

Hence by (2.4), we have (2.3), and inequalities (2.4) and (2.3) are equivalent.

If the constant factor in (2.4) is not the best possible, by the inequality $(T f, g) \leq$ $\|T f\|_{p, w}\|g\|_{q, \widetilde{w}}$ we may get a contradiction that the constant factor in (2.4) is not the best possible.

In the same way, (2.3) and (2.5) are equivalent and the constant factors are the best possible.

Remarks. The results of this paper include many other conclusions which have been published. For instance, in the following we suppose that the integrals in the right of the following inequalities converge to some positive numbers, $\frac{1}{p}+\frac{1}{q}=1$, and one omits the words that the constants factors are the best possible.

1) It is easy to see that for $t=0, l=r, p>1, r=s=2, \lambda=4, H(x, y)=$ $\frac{1}{(x+a y)^{2}(x+a y)^{2}}$, the inequality $(2.1)$ reduces to [9]

$$
\begin{aligned}
& \int_{0}^{\infty} \int_{0}^{\infty} \frac{1}{(x+a y)^{2}(x+a y)^{2}} f(x) g(y) d x d y \\
& <K\left\{\int_{0}^{\infty} x^{-p-1} f^{p}(x) d x\right\}^{\frac{1}{p}}\left\{\int_{0}^{\infty} y^{-q-1} g^{q}(y) d y\right\}^{\frac{1}{q}},
\end{aligned}
$$

where

$$
K=\int_{0}^{\infty} \frac{d t}{(1+a t)^{2}(1+a t)^{2}}=\left\{\begin{array}{l}
\left.\frac{a+b}{(b-a)^{2}} \frac{\ln (b / a)}{b-a}-\frac{2}{a+b}\right], \text { if } a \neq b \\
\frac{1}{6 a^{2}}, \quad \text { if } a=b .
\end{array}\right.
$$


2) Setting $t=0, l=r, p>1, H(x, y)=\frac{|\ln (x / y)|}{(\max \{x, y\})^{\lambda}}(\lambda>0)$, then we have [10]

$$
\begin{aligned}
& \int_{0}^{\infty} \int_{0}^{\infty} \frac{|\ln (x / y)|}{(\max \{x, y\})^{\lambda}} f(x) g(y) d x d y \\
& <\frac{r^{2}+s^{2}}{\lambda^{2}}\left\{\int_{0}^{\infty} x^{p(1-\lambda / s)-1} f^{p}(x) d x\right\}^{\frac{1}{p}}\left\{\int_{0}^{\infty} y^{q(1-\lambda / r)-1} g^{q}(y) d y\right\}^{\frac{1}{q}} .
\end{aligned}
$$

3) Setting $t=0, l=r, p>1, H(x, y)=\frac{|\ln (x / y)|^{\beta}}{(x+y)^{\lambda-\alpha}(\max \{x, y\})^{\alpha}}(\lambda>0, \alpha \in \mathbb{R}, \beta>$ $-1)$, then we have [11]

$$
\begin{aligned}
& \int_{0}^{\infty} \int_{0}^{\infty} \frac{|\ln (x / y)|^{\beta}}{(x+y)^{\lambda-\alpha}(\max \{x, y\})^{\alpha}} f(x) g(y) d x d y \\
& <\Gamma(\beta+1) \Sigma_{k=0}^{\infty}\left(\begin{array}{l}
\alpha-\lambda \\
k
\end{array}\right)\left[\frac{1}{\left(k+\frac{\lambda}{r}\right)^{\beta+1}}+\frac{1}{\left(k+\frac{\lambda}{s}\right)^{\beta+1}}\right] \\
& \times\left\{\int_{0}^{\infty} x^{p(1-\lambda / s)-1} f^{p}(x) d x\right\}^{\frac{1}{p}}\left\{\int_{0}^{\infty} y^{q(1-\lambda / r)-1} g^{q}(y) d y\right\}^{\frac{1}{q}}
\end{aligned}
$$

4) Setting $t=0, l=r=2, p>1, H(x, y)=\frac{\arctan ^{\beta} \sqrt{x^{\lambda} / y^{\lambda}}}{x^{\lambda}+y^{\lambda}}(\lambda>0, \beta>-1)$, then we have [12]

$$
\begin{aligned}
& \int_{0}^{\infty} \int_{0}^{\infty} \frac{\arctan ^{\beta} \sqrt{x^{\lambda} / y^{\lambda}}}{x^{\lambda}+y^{\lambda}} f(x) g(y) d x d y \\
& <\frac{2}{\lambda(\beta+1)}\left(\frac{\pi}{2}\right)^{\beta+1}\left\{\int_{0}^{\infty} x^{p(1-\lambda / 2)-1} f^{p}(x) d x\right\}^{\frac{1}{p}}\left\{\int_{0}^{\infty} y^{q(1-\lambda / 2)-1} g^{q}(y) d y\right\}^{\frac{1}{q}} .
\end{aligned}
$$

5) Setting $t=0, l=r, p>1, H(x, y)=\frac{(\min \{x, y\})^{\lambda}}{|x+y|^{2 \lambda}}, 0<\lambda<\frac{1}{2}$, then we have

$$
\begin{aligned}
\int_{0}^{\infty} \int_{0}^{\infty} \frac{(\min \{x, y\})^{\lambda}}{|x+y|^{2 \lambda}} f(x) g(y) d x d y \\
<\left[B\left(1-2 \lambda,\left(1+\frac{1}{s}\right) \lambda\right)+B\left(1-2 \lambda,\left(1+\frac{1}{r}\right) \lambda\right)\right] \\
\times\left\{\int_{0}^{\infty} x^{p(1-\lambda / r)-1} f^{p}(x) d x\right\}^{\frac{1}{p}}\left\{\int_{0}^{\infty} y^{q(1-\lambda / s)-1} g^{q}(y) d y\right\}^{\frac{1}{q}}
\end{aligned}
$$


$[14]$

6) Setting $t=0, l=r, 1>p>0, H(x, y)=\frac{\ln (x / y)}{x^{\alpha}-y^{\alpha}}, \alpha>0$, then we have

$$
\begin{aligned}
& \int_{0}^{\infty} \int_{0}^{\infty} \frac{\ln (x / y)}{x^{\alpha}-y^{\alpha}} f(x) g(y) d x d y \\
& >\left[\frac{\pi}{\alpha \sin \left(\frac{\pi}{r}\right)}\right]^{2}\left\{\int_{0}^{\infty} x^{p(1-\lambda / s)-1} f^{p}(x) d x\right\}^{\frac{1}{p}}\left\{\int_{0}^{\infty} y^{q(1-\lambda / r)-1} g^{q}(y) d y\right\}^{\frac{1}{q}} .
\end{aligned}
$$

7) Setting

a) $t=1, l=r=p, p>1, \lambda=1, H(x, y)=\frac{1}{|x-y|^{1-\alpha} \min \left\{x^{\alpha}, y^{\alpha}\right\}}, 0<\alpha<\min \left\{\frac{1}{p}, \frac{1}{q}\right\}$ then we have [15]

$$
\begin{aligned}
& \int_{0}^{\infty} \int_{0}^{\infty} \frac{1}{|x-y|^{1-\alpha} \min \left\{x^{\alpha}, y^{\alpha}\right\}} f(x) g(y) d x d y \\
& <\left[B\left(\frac{1}{p}-\alpha, \alpha\right)+B\left(\frac{1}{q}-\alpha, \alpha\right)\right]\left\{\int_{0}^{\infty} f^{p}(x) d x\right\}^{\frac{1}{p}}\left\{\int_{0}^{\infty} g^{q}(y) d y\right\}^{\frac{1}{q}},
\end{aligned}
$$

b) $t=0, \lambda=1, l=p, p>1, H(x, y)=\frac{1}{|x-y|^{1-\alpha} \min \left\{x^{\alpha}, y^{\alpha}\right\}}, 0<\alpha<\min \left\{\frac{1}{p}, \frac{1}{q}\right\}$ then we have [15]

$$
\begin{aligned}
& \int_{0}^{\infty} \int_{0}^{\infty} \frac{1}{|x-y|^{1-\alpha} \min \left\{x^{\alpha}, y^{\alpha}\right\}} f(x) g(y) d x d y \\
& <\left[B\left(\frac{1}{p}-\alpha, \alpha\right)+B\left(\frac{1}{q}-\alpha, \alpha\right)\right]\left\{\int_{0}^{\infty} x^{p-2} f^{p}(x) d x\right\}^{\frac{1}{p}}\left\{\int_{0}^{\infty} y^{q-2} g^{q}(y) d y\right\}^{\frac{1}{q}} .
\end{aligned}
$$

8) Setting $t=1, l=r=q, \lambda=1, p>1, H(x, y)=\frac{|x-y|^{\alpha-1}}{\max \left\{x^{\alpha}, y^{\alpha}\right\}}, \alpha>0$, then [16]

$$
\begin{aligned}
& \int_{0}^{\infty} \int_{0}^{\infty} \frac{|x-y|^{\alpha-1}}{\max \left\{x^{\alpha}, y^{\alpha}\right\}} f(x) g(y) d x d y \\
& <\left[B\left(\frac{1}{p}, \alpha\right)+B\left(\frac{1}{q}, \alpha\right)\right]\left\{\int_{0}^{\infty} f^{p}(x) d x\right\}^{\frac{1}{p}}\left\{\int_{0}^{\infty} g^{q}(y) d y\right\}^{\frac{1}{q}} .
\end{aligned}
$$

9) Setting $t=1, l=r=p, \lambda=1-\alpha, p>1, H(x, y)=\frac{\min \left\{x^{\alpha}, y^{\alpha}\right\}}{\max \{x, y\}}, \alpha \geq 0$, then $[17]$

$$
\int_{0}^{\infty} \int_{0}^{\infty} \frac{\min \left\{x^{\alpha}, y^{\alpha}\right\}}{\max \{x, y\}} f(x) g(y) d x d y<\frac{p q}{1+\alpha}\left\{\int_{0}^{\infty} x^{\alpha} f^{p}(x) d x\right\}^{\frac{1}{p}}\left\{\int_{0}^{\infty} y^{\alpha} g^{q}(y) d y\right\}^{\frac{1}{q}}
$$

10) Setting $t=1, l=r=p, p>1, \lambda>2-\min \{p, q\}, H(x, y)=\frac{\ln (x / y)}{x^{\lambda}-y^{\lambda}}$, then we have [18]

$$
\int_{0}^{\infty} \int_{0}^{\infty} \frac{\ln (x / y)}{x^{\lambda}-y^{\lambda}} f(x) g(y) d x d y
$$


$<\left[\frac{1}{\lambda} B\left(\frac{p+\lambda-2}{p \lambda}, \frac{q+\lambda-2}{q \lambda}\right)\right]^{2}\left\{\int_{0}^{\infty} x^{1-\lambda} f^{p}(x) d x\right\}^{\frac{1}{p}}\left\{\int_{0}^{\infty} y^{1-\lambda} g^{q}(y) d y\right\}^{\frac{1}{q}}$

11) Setting $t=\alpha, l=r=p, 1>p>0, \lambda=\alpha \beta, 2-p<2-q, H(x, y)=$ $\frac{1}{\left(x^{\alpha}+y^{\alpha}\right)^{\beta}}$, then we have[19]

$$
\begin{aligned}
& \int_{0}^{\infty} \int_{0}^{\infty} \frac{1}{\left(x^{\alpha}+y^{\alpha}\right)^{\beta}} f(x) g(y) d x d y \\
& >\frac{1}{\alpha} B\left(\frac{p+\beta-2}{p}, \frac{q+\beta-2}{q}\right)\left\{\int_{0}^{\infty} \frac{\left(x^{1-\alpha} f(x)\right)^{p}}{x^{1+\alpha(\beta-2)}} d x\right\}^{\frac{1}{p}}\left\{\int_{0}^{\infty} \frac{\left(y^{1-\alpha} g(y)\right)^{q}}{y^{1+\alpha(\beta-2)}} d y\right\}^{\frac{1}{q}} .
\end{aligned}
$$

\section{References}

[1] Hardy G. H., Littlewood J. E. and Pólya G., Inequalities, Cambridge University Press, Cambridge, 1952.

[2] Bicheng Yang, On the the norm of an intergal operator and applications, J. Math. Anal. Appl., 2006, 321: 182-192.

[3] Zitian Xie and Zeng Zheng, A Hilbert-type integral inequality whose kernel is a homogeneous form of degree -3, J.Math.Appl., 2008, (339): 324-331.

[4] Zitian Xie and Zeng Zheng, A Hilbert-type inequality with parameters, Natural science Journal of Xiangtan University, 2007, 29(3): 24-28.

[5] Bicheng Yang, A Hilbert-type inequality with a mixed kemel and extensions, Journal of Sichuan Normal University (Natural Science), 2008, 31(3): 281-284.

[6] Zitian Xie and Fang min Zhou, A Generalization of a Hilbert-type inequality with a best constant factor, Journal of Sichuan Normal University (Natural Science), 2009, 32(5): 626-629.

[7] Zitian Xie and Benlu Fu, A new Hilbert-type integral inequality with a best constant factor, J. Wuhan Univ. (Nat.Sci.Ed), 2009, 55(6): 637-640.

[8] Zitian Xie and Xingdong Liu, A new Hilbert-type integral inequality and its reverse, Journal of Henan University (Science Edition), 2009, 39(1)10-13.

[9] Zitian Xie and Bicheng Yang, A new Hilbert-type integral inequality with some parameters and its reverse, Kyungpook Mathe.J., 2008, (48): 93-100.

[10] Bicheng Yang, On a Hilbert-type integral inequality with multiple-parameters, Journal of Southwest China Normal University(Natural Science), 2007, 32(5): 33-38.

[11] Bicheng Yang, A relation to Hilbert's integral inequality and some base Hilbert-type inequalities, Journal of inequalities in pure and applied mathematics, 2008, 9(2), Article 59: 1-8.

[12] Bicheng Yang, A base Hilbert-type integral inequality with the homogeneous kernel of -1-degree and extensions, Journal of Guangong Education Institute, 2008, 28(3): $5-10$. 
[13] Dongmei Xin, On a new Hilbert-type integral inequality, Kyungpook Math.J., 2009, (49): 393-401.

[14] Wuyi Zhong and Bicheng Yang, A reverse Hilbert's type integral inequality with some parameters and the equalent forms, Pure and Applied Mathematics, 2008, 24(2), 401-407.

[15] Bicheng Yang, On a Hilbert-type integral inequality with a parameter, Pure and Applied Mathematics, 2008, 24(3), 489-494.

[16] Bicheng Yang, A new Hilbert-type integral inequality, Journal of Jilin University (Science Edition) 2007, 45(1): 63-67.

[17] Bicheng Yang, A Hilbert-type integral inequality, Journal of Zhejiang University (Science Edition), 2007, 34(2): 121-124.

[18] Bicheng Yang, An extension of the Hilbert's type integral inequality and its applications, J. of Math., (PRC)2007, 27(3): 285-290.

[19] Bicheng Yang, On a reverse Hardy-Hilbert's inequality „Kyungpook Mathe.J., 2007, (47): 411-423.

[20] Zitian Xie and Ju-min Mu Rong, A New Hilbert type Inequality with some parameters, Journal of South China Normal University (Natural Science Edition) 2008, 120(2): 38-42.

[21] Zitian Xie, A reverse Hilbert-type inequality with a best constant Factor, J. Math. Anal. Appl., 2008, 343: 1154-1160.

[22] Jichang Kang, Applied Inequalities, Shangdong Science and Technology press, Jinan, 2004. 